\title{
Direct evaluation of the equilibrium distribution of physical clusters by a grand canonical Monte Carlo simulation
}

\author{
I. Kusaka, Z.-G. Wang, and J. H. Seinfeld \\ Department of Chemical Engineering, California Institute of Technology, Pasadena, California 91125
}

(Received 29 September 1997; accepted 25 November 1997)

\begin{abstract}
A new approach to cluster simulation is developed in the context of nucleation theory. This approach is free of any arbitrariness involved in the definition of a cluster. Instead, it preferentially and automatically generates the physical clusters, defined as the density fluctuations that lead to nucleation, and determines their equilibrium distribution in a single simulation, thereby completely bypassing the computationally expensive free energy evaluation that is necessary in a conventional approach. The validity of the method is demonstrated for a single component system using a model potential for water under several values of supersaturation. (C) 1998 American Institute of Physics. [S0021-9606(98)50509-0]
\end{abstract}

\section{INTRODUCTION}

When a vapor is brought to supersaturation, relaxation occurs toward the more stable liquid phase. The initial stage of this phase transition is the formation of a critical nucleus as a result of spontaneous density fluctuations in the metastable vapor phase. Since not all of the density fluctuations lead to nucleation, Reiss et al. ${ }^{1-6}$ posed a question regarding how to identify a physical cluster, which is defined as a density fluctuation that participates in the nucleation event. Moreover, if nucleation theory is to be formulated in terms of a cluster, as in the classical theory, ${ }^{7}$ its precise characterization is the prerequisite of the theory. One of the quantities of central importance to nucleation theory is the equilibrium cluster size distribution, i.e., the average number of clusters of different sizes per unit volume. Once the distribution is determined, a rate theory can then describe the event of nucleation, ${ }^{7}$ such as its transient or steady state behavior. Since the number density of a given cluster is related to the reversible work to form this cluster in the vapor phase, a cluster simulation in the context of nucleation usually focuses on evaluating the free energy of the clusters.

A cluster simulation is commonly realized by confining a fixed number of molecules, say $i$, in a spherical container of volume $v$ concentric with the center of mass of the $i$ molecules. To the extent that these $i$ molecules actually form a cluster and that its thermodynamic properties are nearly independent of $v$ over a wide range of $v$, Lee et al. ${ }^{8}$ characterized the cluster by its size $i$ alone. We refer to this cluster as the LBA cluster. In an attempt to identify a physical cluster, Reiss et al. ${ }^{1-6}$ characterized the cluster by both $i$ and $v$. The latter is related to the distance from the cluster's center of mass to the nearest ideal gas molecule, which serves as an index to organize the counting procedure in enumerating the configurational space of the entire vapor phase that is regarded as an ideal gas mixture of monomers and clusters of various sizes. As they have pointed out, ${ }^{1-5}$ however, the identification of a physical cluster has to reflect the dynamics of the nucleation process. Thus in their approach, it is the rate theory ${ }^{5}$ that determines whether a particular $i / v$ cluster participates in the nucleation event. Insofar as the cluster size distribution is obtained indirectly from the free energy, which in turn is evaluated through thermodynamic integration, ${ }^{9}$ the simulation has to be carried out at many values of temperature for each cluster size $i$ or each pair of values of $i$ and $v$ in the case of the $i / v$ cluster. For this reason, the $i / v$-cluster was also studied by computationally less demanding density functional theory. ${ }^{10}$

In this work, we present a new approach to the problem which directly implements the stochastic evolution of a physical cluster in the form of a grand canonical Monte Carlo simulation. ${ }^{9}$ Our approach is conceptually simpler than the $i / v$ cluster and offers several attractive features. The simulation preferentially generates the physical clusters during the course of the simulation without any prior knowledge regarding the details of their identity. Their equilibrium distribution is, at least in principle, directly determined from a single simulation, which permits one to completely bypass the expensive free energy evaluation. The grand canonical Monte Carlo simulation presents an additional advantage of efficiently sampling the different relevant configurations even for a cluster of highly associative molecules. Finally, the approach maintains its simplicity regardless of the complexity of the intermolecular interaction arising, for example, from the presence of a molecular polarizability or three-body potentials.

The remainder of the paper is organized as follows. In Sec. II A, we review a conceptual aspect in formulating nucleation theory, which clarifies the context in which a physical cluster should be introduced into the theory. Details of the method to identify and characterize the physical cluster are given in Sec. II B followed by Sec. II C which describes how to evaluate, from a single simulation, the equilibrium distribution of the physical clusters. The method is applied in Sec. III to water using the SPC/E model ${ }^{11}$ to illustrate its utility. The paper then concludes in Sec. IV with a brief discussion on the implications of our work. 


\section{THEORY}

\section{A. General concept}

We first review a conceptual aspect in describing a nucleation process. In principle, a fundamental microscopic theory can describe the time evolution of the density fluctuations that occur spontaneously in a metastable state and eventually lead to the formation of a new phase. In the framework of classical mechanics, for example, one determines the phase space trajectory that brings the system from the metastable phase to the more stable phase. When the average is taken over all possible initial microstates consistent with the metastable phase, one obtains a statistical description of the phase transition. In the search for a macroscopic description of the process, one introduces a coarse graining into the microscopic theory, namely a number of microstates are grouped together as one entity, which we temporarily call a macrostate, and the deterministic elements in the fundamental microscopic theory are replaced by a probabilistic description providing the transition rates between these macrostates, each of which is now characterized by average properties of the microstates contributing to it. Reiss et al. ${ }^{1-5}$ addressed this aspect as an "inversion of the order of averaging." It is by no means a peculiarity in nucleation theory, rather it is a central theme of statistical physics. The method is valid if, for example, the system can be divided into many statistically independent small parts and an experiment is not sufficiently sensitive to probe beyond the average behavior of these small parts.

Needless to say, it is a difficult task to explicitly carry out the approach just mentioned. However, nucleation theory is concerned with the initial stage of the phase transition, i.e., formation of a critical nucleus as a result of the spontaneous density fluctuations occurring in the parent phase in metastable equilibrium. Thus equilibrium statistical mechanics should suffice in identifying the microstates relevant in nucleation. Consequently, one can introduce the macrostates through the coarse graining of the configurational space of the system constrained in the metastable state. It remains to specify the statistical ensemble most suitable in identifying a physical cluster. In the present context, a physical cluster is defined as a density fluctuation in the metastable phase that leads to nucleation. ${ }^{1-6}$ If the entire vapor phase is taken as a system, the coarse graining itself does not allow one to identify the physical cluster, for a macrostate introduced in this procedure represents a group of points in the configurational space of the entire vapor. Even though it is still possible to define a cluster, it merely serves as a counting procedure in enumerating the configurational space. Apparently, the definition is not unique since one can organize the counting in an arbitrary fashion. Any arbitrariness in defining the cluster must be removed by the rate theory appropriate for that definition. This implies that neither the cluster thus defined nor the rate theory is completely free of a nonphysical aspect. In developing a molecular level theory, however, we demand that the cluster introduced into the theory or the rate theory taken separately be subject to a direct physical interpretation. Thus we take a system so that the identification of a physical cluster and its characterization in terms of a macrostate are accomplished as a result of the coarse graining. In this work, we restrict ourselves to the case of vapor to liquid homogeneous nucleation in a single component system. The formalism developed here can be extended to a binary system or heterogeneous nucleation, for example.

\section{B. Identification of a physical cluster}

Suppose that the entire vapor phase of volume $V_{\text {tot }}$ is divided into small cells of equal volume $V$ and assume that $V$ satisfies the following two conditions. ${ }^{12}$ On one hand, $V$ is sufficiently macroscopic in the sense that these cells can be regarded as statistically independent, which permits one to describe the nucleation process in the entire vapor by focusing on a single cell of volume $V$. In other words, performing an experimental measurement on the whole vapor phase is equivalent to taking an ensemble average on one of the cells. The appropriate statistical ensemble is a grand canonical ensemble. ${ }^{13}$ On the other hand, $V$ is small enough that the probability of finding more than one uncorrelated density fluctuation that participates in the nucleation process at any instant is negligible, which implies that there is at most one cluster in the cell. Thus a proper coarse graining of the configurational space of the grand canonical ensemble should lead to an identification of a physical cluster. The appropriate partition function for one such cell is

$$
\Xi(\beta, V, z)=\sum_{N=0}^{N_{\text {cut }}} \frac{z^{N}}{N !} \int d\{N\} e^{-\beta U_{N},}
$$

where $\beta=\left(k_{B} T\right)^{-1}$ with $k_{B}$ and $T$ being the Boltzmann constant and the absolute temperature, respectively. $z$ is the fugacity of the molecule and $N$ is the total number of molecules in the system, whose translational and orientation coordinates are collectively denoted by $\{N\}$. The total potential energy of the system is denoted by $U_{N}$. The summation with respect to $N$ is bounded by $N_{\text {cut }}$ to constrain the system to the metastable equilibrium. Equation (1) suggests that one can classify the microstates by $N$ alone or $N$ and $U_{N}$. Complication arises, however, since $V$ is macroscopic and most of the fluctuations in $N$ and $U_{N}$ have very little to do with the nucleation process. Thus, among the various microstates consistent with a given value of $N$ and a given interval of $U_{N}$, only a small fraction of them actually participate in the nucleation event and hence contain a physical cluster. In the language of Sec. II A, this means that a finer coarse graining than achieved by $N$ or $N$ and $U_{N}$ is required to identify the microstates containing a physical cluster.

A conventional approach to identify the relevant microstates is to a priori define a set of clusters from which the physical clusters are isolated. For example, Band ${ }^{14,15}$ and Stillinger ${ }^{16}$ defined a cluster such that a molecule is considered to be a part of it if the distance between the molecule and at least one of the molecules of the cluster is less than a certain cutoff distance. In the LBA cluster, ${ }^{8}$ every molecule in the system of volume $v \ll V$ is regarded as a part of the cluster. When either the cutoff distance or $v$ is chosen so that the thermodynamic properties of the cluster are insensitive to it, the resulting cluster is identified as the physical cluster. In the $i / v$ cluster, ${ }^{1-6}$ every molecule in the system of volume $v$ 
is also regarded as a part of the cluster. The $i / v$ cluster differs from the LBA cluster in that the physical clusters are isolated from the various $i / v$ clusters through an appropriate rate theory. ${ }^{5}$ The assumption implicit in these approaches is that the arbitrarily defined clusters form a superset of the physical clusters. It is not even obvious, however, that all the relevant microstates are distributed primarily among the clusters that are to be isolated as physical or that all the irrelevant microstates are distributed primarily among the clusters that are to be discarded as nonphysical. For example, consider two microstates: one with an $i / v$ cluster and the other with an $i / v^{\prime}$ cluster, and suppose that the configurations of molecules inside $v$ and $v^{\prime}$ are identical and that these two clusters differ only by their values of $v$, namely by the locations of the nearest ideal gas molecules. If the $i / v$ cluster is a physical one and $v \ll v^{\prime}$, the $i / v^{\prime}$ cluster is most likely a nonphysical one, for the assumed configuration in the $i / v^{\prime}$ cluster is unfavorable because of the translational entropy of the molecules and may not be sampled at all during a simulation of a finite length of time. Artifacts of this kind would be removed, if possible at all, only by an intractably complicated rate theory.

One must realize that whether or not a given microstate contains a physical cluster, and hence is relevant in a nucleation event, is completely determined by the system itself. Thus it is most straightforward to directly isolate the physical clusters from the entire microstates accessible to the system rather than indirectly by means of an arbitrarily defined cluster. The remaining task is simply to devise an effective simulation method for this purpose in such a way that no prior knowledge is required as to the detailed identity of the physical clusters. Let us first define the excess number of molecules $N_{c}$ and the excess potential energy $U_{c}$ by

$$
\begin{aligned}
& N_{c} \equiv N-n_{v} V, \\
& U_{c} \equiv U_{N}-u_{v} V,
\end{aligned}
$$

where $n_{v}$ and $u_{v}$ are the number density of molecules and the average potential energy per unit volume in the vapor phase, respectively. Note that $N_{c}$ is, in general, not an integer or necessarily positive. During a simulation, $N$ and $U_{N}$, hence $N_{c}$ and $U_{c}$, fluctuate. For a macroscopic $V$, these fluctuations are caused primarily by those due to each of the vapor molecules. Fluctuations of this kind are undesirable in identifying the physical clusters since they have very little to do with the nucleation process. In a grand canonical ensemble, however, their effect on $N_{c}$ or $U_{c}$ can be made negligible by decreasing the volume until it satisfies

$$
n_{v} V \ll 1 \text {. }
$$

In this limit, the system contains, on average, no vapor molecules and thus the simulation preferentially generates the microstates containing a physical cluster. In fact, the probability of finding at least one vapor molecule in the system is, assuming the ideal gas behavior of the vapor phase, given by $1-e^{-n_{v} V} \approx n_{v} V$, which is negligible as a result of Eq. (3). To achieve the coarse grained description, one can characterize the physical cluster by the excess quantities $N_{c}$ and $U_{c}$ since the dominant contribution to them now arises from the presence of the cluster. A more detailed characterization of the cluster is clearly possible. In what follows, however, we focus solely on $N_{c}$ since the inclusion of $U_{c}$ or some other quantities does not affect the theoretical development given below. We refer to the physical cluster characterized in this manner as an $N_{c}$-cluster.

In view of Eq. (3), we may redefine $N_{c}$ by

$$
N_{c} \equiv N \text {, }
$$

so that $N_{c}$ is a non-negative integer. In short, our approach is to perform a grand canonical Monte Carlo simulation ${ }^{9}$ on the system of microscopic volume $V$ satisfying Eq. (3) allowing the number of molecules $N_{c}$ to fluctuate from 0 to $N_{\text {cut }}$. All of the molecules found inside the system at a given instant are regarded as forming a physical cluster. To constrain the system to the metastable equilibrium, while still sampling a critical nucleus, $N_{\text {cut }}$ introduced in Eq. (1) must be chosen to slightly exceed the size of the critical nucleus. Among the microstates contributing to the $N_{c}$-cluster, there are undoubtedly configurations in which some of the molecules are more properly regarded as part of the vapor. The extent to which such configurations contribute to thermodynamic properties of the $N_{c}$-cluster, and hence the transition rates between the various $N_{c}$-clusters clearly depends on $V$. This $V$ dependence is neither an artifact nor an arbitrariness of the theory, rather the magnitude of the vapor contributions reflects the focus of our coarse grained description of the nucleation phenomenon. Because of Eq. (3), however, the vapor contribution is on average negligible. Consequently, the volume dependence is expected to be negligible as well and we shall not dwell upon this issue any further.

Some words on $V$ are in order. Clearly, $V$ has to be larger than the spatial extent of a physical cluster in it. That the system is microscopic does not affect the applicability of the statistical mechanical description. It is sufficient to assume a weak coupling between the system and its surroundings. ${ }^{13}$ Both conditions are trivially satisfied in the case of vapor to liquid nucleation, in which the molar volume in the vapor phase is considerably larger than the physical dimension of the cluster and the interaction between the vapor molecules and a cluster can be ignored. If, on the other hand, $V$ cannot be chosen to satisfy Eq. (3), the very concept of cluster is no longer relevant in describing nucleation, for the correlation between the system inside $V$ and the surrounding is appreciable in this case. In other words, our method is applicable whenever the concept of cluster is relevant in nucleation and vice versa, implying that clusters identified in our method are in fact physical clusters.

\section{The equilibrium cluster size distribution}

Besides providing a natural way to identify and characterize the physical clusters, the grand canonical ensemble allows one to obtain the equilibrium cluster size distribution directly from a single simulation without an expensive free energy evaluation. Since the distribution is of central importance in nucleation theory, we examine this possibility in detail.

To derive the expression for the equilibrium cluster size distribution $c\left(N_{c}\right)$, suppose that the whole vapor phase of 
volume $V_{\text {tot }}$ is divided into cells of equal volume $V$, where $V$ satisfies Eq. (3). Because of Eq. (3), most of the cells contain no molecules at all and those containing an $N_{c}$-cluster, including $N_{c}=1$, are on average spatially distant. Thus as we discussed at the end of Sec. II B, each cell is only weakly coupled with its surrounding cells and one can assume that the cells are all statistically independent. Consequently, the average number of the $N_{c}$-cluster inside the entire vapor of volume $V_{\text {tot }}$ is given by

$$
\frac{V_{\mathrm{tot}} \Xi_{c}\left(\beta, V, z ; N_{c}\right)}{V \Xi(\beta, V, z)},
$$

where $\Xi_{c}$ is the term for which $N=N_{c}$ in the grand canonical partition function $\Xi$ :

$$
\Xi_{c}\left(\beta, V, z ; N_{c}\right) \equiv \frac{z^{N_{c}}}{N_{c} !} \int d\left\{N_{c}\right\} e^{-\beta U_{N_{c}} .}
$$

Equation (5) can be rationalized as follows: $V_{\text {tot }} / V$ is the total number of cells, while

$$
p\left(N_{c}\right) \equiv \frac{\Xi_{c}\left(\beta, V, z ; N_{c}\right)}{\Xi(\beta, V, z)}
$$

is the normalized probability of finding an $N_{c}$-cluster in a given cell. Dividing the expression Eq. (5) by $V_{\text {tot }}$, we obtain the desired number density:

$$
c\left(N_{c}\right)=\frac{1}{V} p\left(N_{c}\right) .
$$

Since the cluster can be formed anywhere in the volume $V$, we have

$$
p\left(N_{c}\right) \sim V,
$$

provided that care is taken to avoid the surface effect. As we shall see shortly, the implementation of this condition in a simulation is trivial. Thus $c\left(N_{c}\right)$ given by Eq. (8) is independent of $V$ as it should be.

In principle, one can determine the normalized probability $p\left(N_{c}\right)$ from a single simulation simply by counting the number of events in which $N_{c}$ molecules are found in the system. Because of the condition Eq. (3), however, the system contains no molecules at all for most of the time and the states with $N_{c} \geqslant 1$ will be hardly sampled. To avoid this, we perform the simulation by fixing one molecule at the center. Provided that the boundary of the system is far from the molecules forming a cluster, the problem associated with the surface effect mentioned above is also resolved. Namely, the volume dependency Eq. (9) is rigorously obtained if integration with respect to the coordinates of the molecule thus fixed is performed analytically by ignoring the surface effect.

Since the precise size of the critical nucleus is not known prior to a simulation, the appropriate value of $N_{\text {cut }}$ is also unknown. It is then convenient to perform a simulation by allowing $N_{c}$ to fluctuate from unity to $N_{\max }$ chosen to be sufficiently large compared to the expected value of $N_{\text {cut }}$. Clearly, the normalized probability $p_{s}\left(N_{c}\right)$ obtained from the simulation differs from $p\left(N_{c}\right)$, since the former is normalized in the interval $\left[1, N_{\max }\right]$, while the latter must be nor- malized in $\left[0, N_{\text {cut }}\right]$. To express $p\left(N_{c}\right)$ in terms of $p_{s}\left(N_{c}\right)$, we first note that the ratio $p_{s}\left(N_{c}\right) / p\left(N_{c}\right)$ is a common constant for $N_{c}=1, \ldots, N_{\text {cut }}$. In fact,

$$
\frac{p_{s}\left(N_{c}\right)}{p\left(N_{c}\right)}=\frac{\Xi_{c}\left(N_{c}\right) / \Xi^{\prime}}{\Xi_{c}\left(N_{c}\right) / \Xi},
$$

where $\Xi^{\prime}$ is a grand canonical partition function of the system when $N_{c}$ is constrained to $N_{c}=1, \ldots, N_{\max }$. Next, note that the ratio $p(0) / p(1)$ can be obtained analytically if the interaction between the system and the surroundings is ignored:

$$
\frac{p(0)}{p(1)}=\frac{1}{z \Omega V},
$$

where $\Omega$ arises from the integration with respect to the orientational coordinates. Equations (10) and (11) can be used with the normalization requirement of $p\left(N_{c}\right)$ to obtain

$$
\begin{aligned}
& p(0)=\frac{p_{s}(1)}{p_{s}(1)+z \Omega V \sigma}, \\
& p\left(N_{c}\right)=\frac{z \Omega V p_{s}\left(N_{c}\right)}{p_{s}(1)+z \Omega V \sigma} \quad\left(N_{c}=1, \ldots, N_{\mathrm{cut}}\right),
\end{aligned}
$$

where we define

$$
\sigma \equiv \sum_{N_{c}=1}^{N_{\text {cut }}} p_{s}\left(N_{c}\right) .
$$

The equilibrium distribution $c\left(N_{c}\right)$ immediately follows from Eq. (8). The second of Eq. (12) satisfies Eq. (9). To see this, note that $p_{s}\left(N_{c}\right)$ is independent of $V$ since both $\Xi_{c}$ and $\Xi^{\prime}$ are proportional to $V$. Upon ignoring the terms of the second order in $z \Omega V$ or higher, Eq. (12) yields Eq. (9).

The simulation becomes impractical as the free energy barrier of nucleation exceeds several $k_{B} T$ since clusters around the critical size are hardly reached. To overcome this difficulty, one can simply perform a series of simulations allowing $N_{c}$ to fluctuate in the intervals $\left[1, N_{1}\right]$, $\left[N_{1}, N_{2}\right], \ldots,\left[N_{n}, N_{\max }\right]$. The probability $p_{s}\left(N_{c}\right)$ readily follows by demanding its continuity. This is an example of the umbrella sampling. ${ }^{17}$ The validity of this approach depends on the assumption that $N_{i}$ molecules form a cluster in the simulation constrained in $\left[N_{i}, N_{i+1}\right]$, which is reasonable since the system, when viewed as a closed one with $N_{i}$ molecules inside, is at least $N_{i}$-fold more supersaturated than the vapor phase as is seen from Eq. (3):

$$
\frac{N_{i}}{V} \gg N_{i} n_{v} .
$$

The fact that $N_{c}$ is constrained in the interval $\left[N_{i}, N_{i+1}\right]$ does not imply that the same set of $N_{i}$ molecules remains in the system as if they were forming a core on which other molecules condense. Instead, any molecule in the system are subject to a trial annihilation in the grand canonical Monte Carlo simulation as far as $N_{c}$ exceeds $N_{i}$. This is especially important to sample efficiently all the relevant configurations of a cluster of highly associative molecules.

The time scale for a cluster to reach internal mechanical equilibrium is many orders of magnitude shorter than that for 
the cluster to exchange a molecule with the vapor phase. Therefore, it is a common practice to assume that the configurational integral of an $N_{c}$-cluster is independent of the fugacity. Under this assumption, the results obtained at fugacity $z^{\prime}$ can be used to estimate $c\left(N_{c}\right)$ at a different fugacity $z^{\prime \prime}$. To see this, it is sufficient to note that

$$
p_{s}\left(z^{\prime \prime}, N_{c}\right)=\frac{\Xi^{\prime}\left(z^{\prime}\right)}{\Xi^{\prime}\left(z^{\prime \prime}\right)}\left(\frac{z^{\prime \prime}}{z^{\prime}}\right)^{N_{c}} p_{s}\left(z^{\prime}, N_{c}\right),
$$

substitution of which in Eq. (12) with $z$ replaced by $z^{\prime \prime}$ in the latter reveals that the unknown constant $\Xi^{\prime}\left(z^{\prime}\right) / \Xi^{\prime}\left(z^{\prime \prime}\right)$ cancels out. It should be noted here that the assumption leading to Eq. (15) is not valid if the $N_{c}$-cluster has more than one conformational isomer that cannot establish a chemical equilibrium among themselves within the time period required for the cluster to change its size. In fact, a change in the fugacity affects the condensation rate of a vapor molecule, while having a minimal effect, if at all, on the evaporation rate, which differs from one isomer to another. Consequently, the probability distribution of isomers accounted for under the $N_{c}$-cluster, and hence the thermodynamic properties of the $N_{c}$-cluster, depends on the fugacity. In this case, one has to perform a separate simulation for each value of the fugacity.

Finally, we address a consistency issue. For simplicity, we assume that the vapor phase can be regarded as an ideal gas, for which $z \Omega=n_{v}$. Strictly speaking, the 1-cluster cannot be identified with a vapor monomer, since the former excludes other molecules from $V$ because of the approximate definition Eq. (4) of $N_{c}$, while the latter does not under the ideal gas approximation. In fact, one can easily show that $c(1) \neq n_{v}: \Xi_{c}$ in Eq. (6) can be evaluated to be $n_{v} V$ for the 1-cluster, while for an ideal gas

$$
\Xi=e^{n_{v} V},
$$

yielding

$$
c(1)=n_{v} e^{-n_{v} V} .
$$

The factor of $e^{-n_{v} V}$ is a work term arising from the volume exclusion just mentioned. However, this distinction is completely insignificant since $e^{-n_{v} V} \approx 1$. Alternatively, one can consistently recover the monomer concentration by setting $V=0$ in Eq. (17).

\section{An alternative interpretation}

We have assumed in Sec. II B that the physical clusters, namely the density fluctuations that participate in the nucleation event, can be identified with the density fluctuations other than those due to each of the vapor molecules. The validity of this assumption can be addressed only through an explicit formulation of the approach discussed in Sec. II A. Given the intractability of the formulation, however, it is of interest to present a heuristic argument to motivate our grand canonical Monte Carlo simulation from an alternative point of view.

Let us focus on an arbitrarily chosen monomer in the vapor phase and define an open system of volume $V$ centered at the monomer. The volume $V$ is taken to be sufficiently small compared to the molar volume of the vapor molecules:

$$
V \ll \frac{1}{n_{v}},
$$

which is nothing more than Eq. (3). If the stochastic evolution of the system is followed throughout the entire nucleation process, one would find, for most of the cases, that the system contains the same monomer alone even after nucleation took place. However, if the monomer we chose was the successful one, we find that it acquires other vapor molecules, which then form a cluster. If nucleation process is a formation of a cluster of this kind as is pictured in the classical nucleation theory, but with a sufficient number of molecules to reach a critical size, the physical clusters are identified simply by following the stochastic evolution of a system that contains a successful monomer. Provided that this stochastic evolution can be described as a Markov process, a monomer can be made to be a successful one by employing a sufficiently high frequency of the trial creation and annihilation of molecules in the system. This is legitimate since a fundamental property of the Markov process guarantees that the limiting distribution of the Markov process, such as the statistical weight of each microstates and $p_{s}\left(N_{c}\right)$, is independent of the frequency, as long as microscopic reversibility is satisfied by the transition rates between microstates. ${ }^{9}$ Our grand canonical Monte Carlo simulation can be viewed as a straightforward implementation of this idea. Conceptually, however, the point of view taken in Sec. II B was preferred since it highlights the subtle difference between our method of identifying the physical clusters and the conventional ones that require an a priori definition of clusters and the necessity of the term $N_{c}=0$ in normalizing $p\left(N_{c}\right)$ is readily understandable.

Finally, we note that the physical clusters generated in the simulation are consistent with an intuitive definition of clusters. To see this, note that, in our simulation, $N_{c}$ is always larger than $n_{v} V \ll 1$, the average number of molecules in $V$ when filled with the uniform vapor. Thus, on average, any attempted Monte Carlo move to create a molecule in the system will be accepted with higher probability if the newly

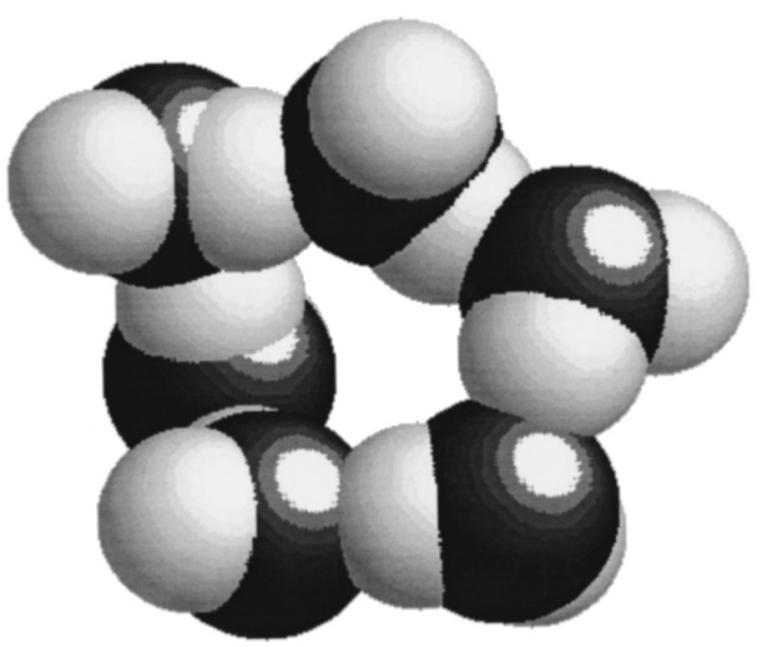

FIG. 1. A snapshot of a 6-cluster forming a cyclic hexamer. At $T$ $=298.15 \mathrm{~K}$ and $z \Omega=0.1 \times 10^{-5} \AA^{-3} . N_{c}$ is confined in the interval $[6,10]$. 
TABLE I. Conditions of the simulation.

\begin{tabular}{cccc}
\hline \hline No. & $z \Omega\left[\AA^{-3}\right]$ & $n_{v} V^{\mathrm{a}}$ & $N_{i}^{\mathrm{b}}$ \\
\hline S1 & $0.23 \times 10^{-5}$ & $0.325 \times 10^{-1}$ & 1,16 \\
S2 & $0.16 \times 10^{-5}$ & $0.226 \times 10^{-1}$ & $1,4,8,20,26$ \\
S3 & $0.12 \times 10^{-5}$ & $0.170 \times 10^{-1}$ & $1,3,7,11,16,22,30,38$ \\
S4 & $0.1 \times 10^{-5}$ & $0.141 \times 10^{-1}$ & $1,3,6,10,16,24,32,40,44$ \\
S5 & $0.8 \times 10^{-6}$ & $0.113 \times 10^{-1}$ & $1,3,6,9,12,16,20,28,36,44,50,55$ \\
\hline \hline
\end{tabular}

ander the ideal gas approximation, $n_{v}=z \Omega$.

${ }^{\mathrm{b}}$ End values of $N_{c}$ in the umbrella sampling.

created molecule interacts more favorably with the rest of the molecules, while as soon as a molecule evaporates from the cluster, it is more likely to be removed from the system upon its trial annihilation.

\section{APPLICATION TO SPC/E WATER}

As an illustration, we applied the present method to water using the SPC/E model, ${ }^{11}$ which is a three interaction sites model without any polarizability known to reproduce some of the bulk liquid properties of water. It should be noted that the interaction potential in a small object such as a cluster and that in the bulk phase can be quite different. ${ }^{18}$ Thus the model may not be accurate for simulating the properties of clusters. We shall not pursue the issue here. Instead, we stress that more realistic model potentials can be employed without any modification to the theory.

We carried out the simulation at $T=298.15 \mathrm{~K}$ for several values of the fugacity. The system is taken as a spherical container of radius $15 \AA$. Other details of the simulation conditions are summarized in Table I. We sampled the value of $N_{c}$ once every $10^{2}$ Monte Carlo steps and performed each simulation until each $N_{c}$-cluster was sampled about $10^{4}$ times. This translates to about $10^{6}$ Monte Carlo steps for each $N_{c}$-cluster. During one Monte Carlo step, translation and rotation is made on average once on every molecule in the system, except for that at the center which undergoes rotation only. Care must be taken to ensure the microscopic

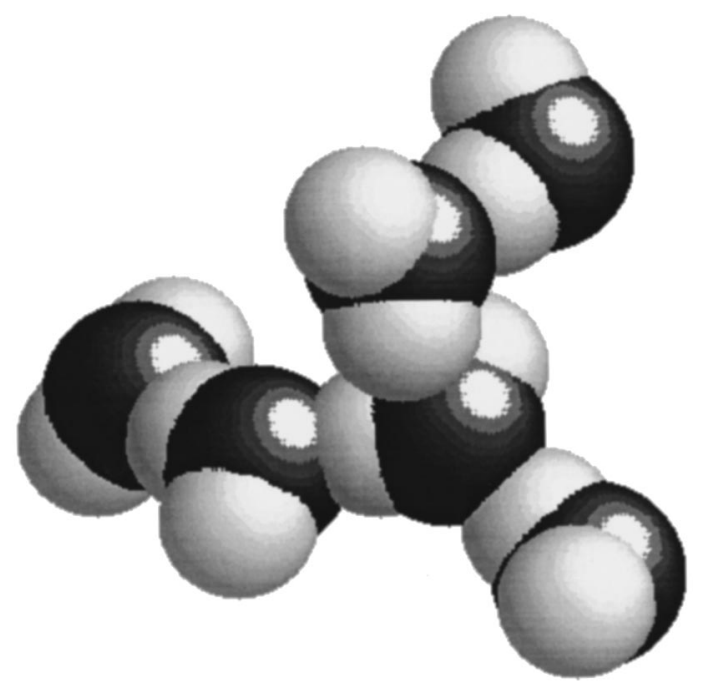

FIG. 2. A snapshot of a 6-cluster. At $T=298.15 \mathrm{~K}$ and $z \Omega=0.1$ $\times 10^{-5} \AA^{-3} . N_{c}$ is confined in the interval $[6,10]$. reversibility upon a trial creation or annihilation of a molecule. Thus during each Monte Carlo step, either trial creation or annihilation of a molecule is performed with equal probability. When $N_{c}$ is at its lower bound, the trial annihilation is rejected with certainty. Likewise for a trial creation when $N_{c}$ is at the upper bound.

Figures $1-5$ show snapshots ${ }^{19}$ taken rather arbitrarily from the simulation. The compact configuration shown in each of the figures clearly qualifies as a cluster. Extensive hydrogen bond network is also observed. Comparison of Figs. 1-3 reveals that very different configurations are sampled even in this highly associative substance, showing a clear advantage of the grand canonical simulation.

The equilibrium cluster size distribution is shown in Fig. 6. For simplicity, we have set $N_{\text {cut }}=N_{\max }$ in Eq. (13). Since we are to describe the event of nucleation as a stochastic evolution of an $N_{c}$-cluster, the relevant reversible work $W^{\text {rev }}\left(N_{c}\right)$ to form this $N_{c}$-cluster is related to its probability $p\left(N_{c}\right)$ or the concentration $c\left(N_{c}\right)$. In this work, we have taken the point of view that a cluster is the density fluctuation in the vapor confined in the metastable state. The reversible work $W^{\mathrm{rev}}\left(N_{c}\right)$ appropriate for this fluctuation picture is

$$
\beta W^{\mathrm{rev}}\left(N_{c}\right) \equiv-\log p\left(N_{c}\right) .
$$

In classical theory, the $N_{c}$-cluster is regarded as a product of the reaction

$$
N_{c} X \rightleftharpoons X_{N_{c}}
$$

where $X$ denotes a monomer. In this reaction picture, $W^{\text {rev }}\left(N_{c}\right)$ is more properly defined by

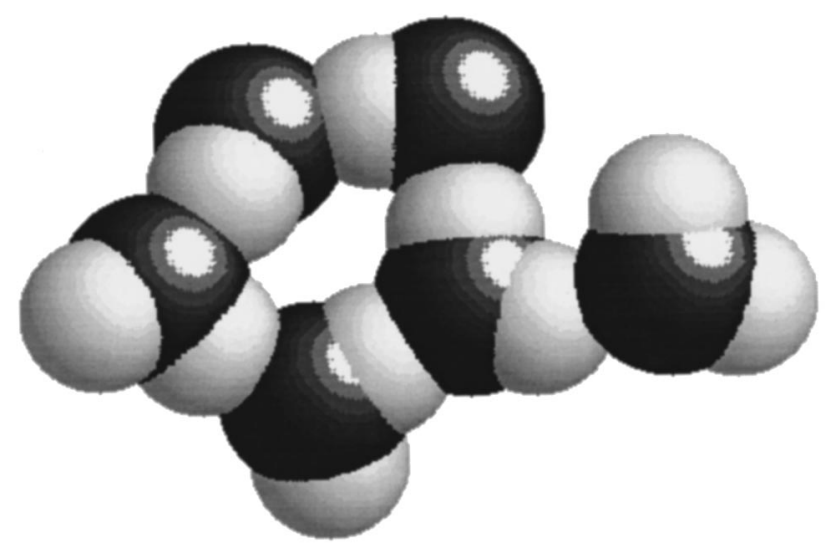

FIG. 3. A snapshot of a 6-cluster as a cyclic pentamer with an additional molecule. At $T=298.15 \mathrm{~K}$ and $z \Omega=0.1 \times 10^{-5} \AA^{-3} . N_{c}$ is confined in the interval $[6,10]$. 


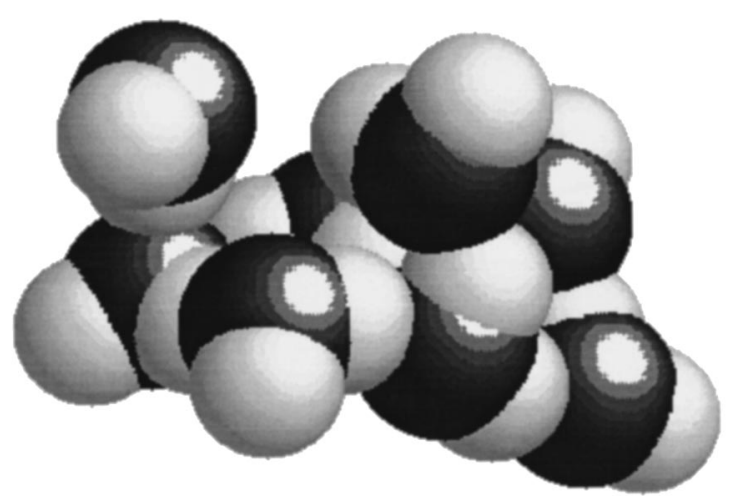

FIG. 4. A snapshot of an 8-cluster. At $T=298.15 \mathrm{~K}$ and $z \Omega=0.1$ $\times 10^{-5} \AA^{-3} . N_{c}$ is confined in the interval $[6,10]$.

$$
\beta W^{\mathrm{rev}}\left(N_{c}\right) \equiv-\log \frac{c\left(N_{c}\right)}{n_{v}} .
$$

The appearance of $n_{v}$ is reasonable since the formation of the $N_{c}$-cluster starts from a monomer. In the case of vapor to liquid nucleation, both the fluctuation picture and the reaction picture appear quite reasonable. Hence, we expect that the rate theory appropriate for the fluctuation picture and that for the reaction picture will yield a consistent description of the nucleation phenomenon. In fact, $c\left(N_{c}\right)$ is independent of which picture is employed. In the following, we focus on $\beta W^{\text {rev }}\left(N_{c}\right)$ defined by Eq. (21), which is shown in Fig. 7. For high enough values of the fugacity, $\beta W^{\mathrm{rev}}(3)$ is found to be a local maximum, which presumably is due to the inability to form a stable hydrogen bond network in the 3-cluster caused by the lack of the polarizability in the model potential. Although this result is most likely an artifact of the model potential, we note that such a nontrivial detail is easily captured in the present approach.

To assess the validity of the assumption implicit in Eq. (15), we compare $\beta W^{\mathrm{rev}}\left(z, N_{c}\right)$ at $z \Omega=0.1 \times 10^{-5} \AA^{-3}$ that is obtained directly from simulation at this value of the

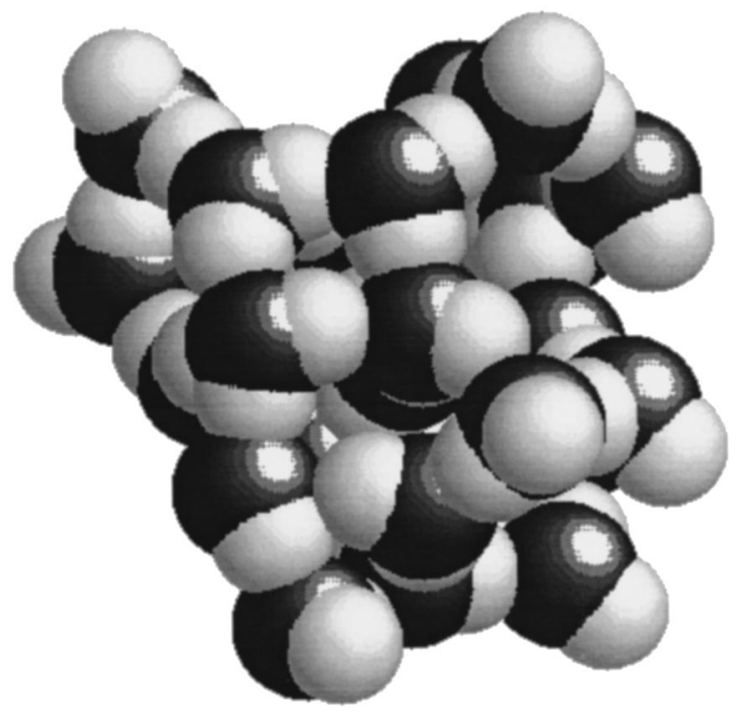

FIG. 5. A snapshot of a 25-cluster. At $T=298.15 \mathrm{~K}$ and $z \Omega=0.1$ $\times 10^{-5} \AA^{-3} . N_{c}$ is confined in the interval [24, 32].

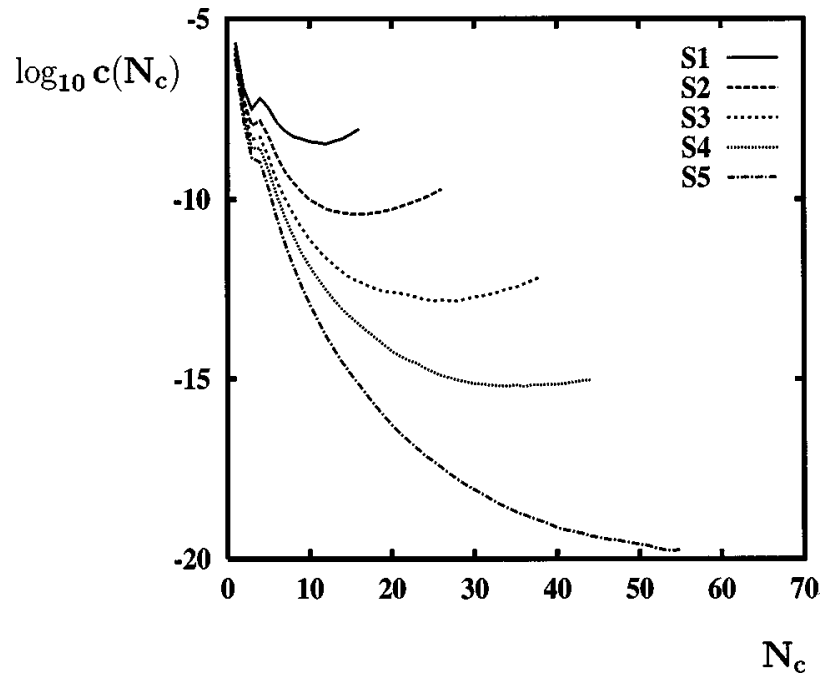

FIG. 6. Cluster size distribution at $T=298.15 \mathrm{~K} . c\left(N_{c}\right)$ is in $\AA^{-3}$. The conditions of simulation for $\mathrm{S} 1, \ldots, \mathrm{S} 5$ are given in Table I.

fugacity with $\beta W^{\text {rev }}\left(z, N_{c}\right)$ obtained through Eq. (15) using $p_{s}\left(z^{\prime}, N_{c}\right)$ for other values of the fugacity $z^{\prime}$, where in Eq. (15) we set $z^{\prime \prime}=z$. The result is shown in Fig. 8 and indicates that the configurational integral, and hence the Helmholtz free energy, of an $N_{c}$-cluster is nearly independent of the value of the fugacity. The result has several important implications. First of all, this is the first example in which the fugacity independence of the thermodynamic properties of a cluster is actually demonstrated rather than simply assumed. Second, if one assumes this fugacity independence from the outset, then the agreement among various simulations provides an independent check that the configurational integral is properly evaluated in each simulation. This is rather remarkable since $10^{6}$ Monte Carlo steps are hardly enough to achieve this kind of convergence for water if a canonical ensemble is employed. Next, one can significantly reduce the computational effort. Namely, when a simulation is performed at a certain value of the fugacity, the result can be

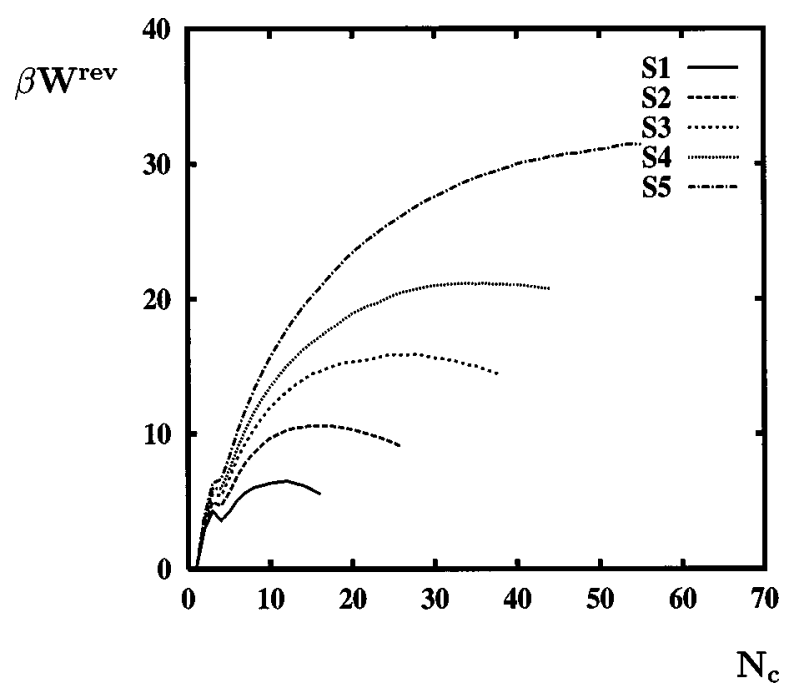

FIG. 7. The reversible work of cluster formation at $T=298.15 \mathrm{~K}$. The conditions of simulation for $\mathrm{S} 1, \ldots, \mathrm{S} 5$ are given in Table I. 


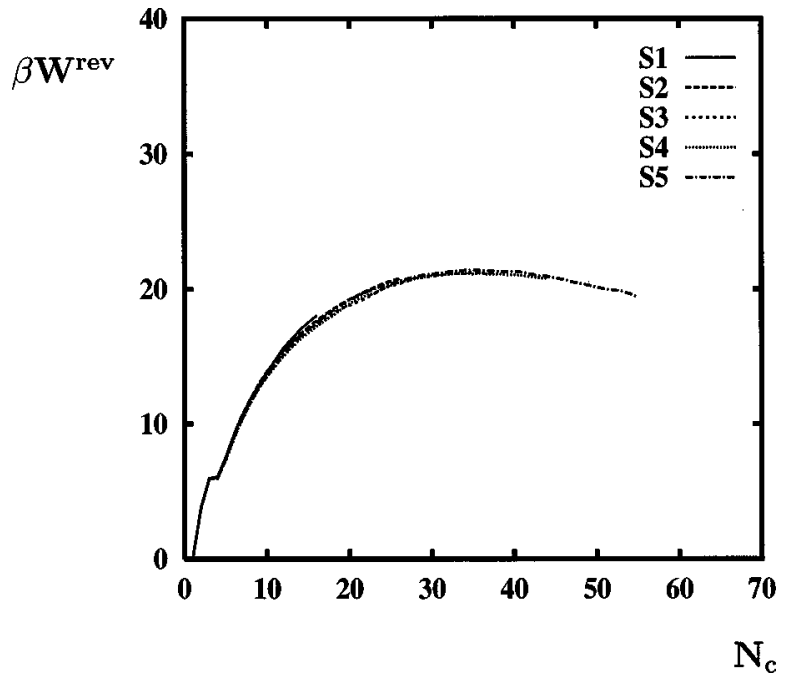

FIG. 8. Comparison of the reversible work of cluster formation at $T$ $=298.15 \mathrm{~K}$ and $z \Omega=0.1 \times 10^{-5} \AA^{-3}$ obtained directly at this value of $z \Omega$ (S4) with the same quantity obtained through Eq. (15) by using other values of $z \Omega$ (S1, S2, S3, S5). The conditions of simulation for S1,..,S5 are given in Table I.

used to calculate the reversible work at any value of the fugacity, provided that $N_{\max }$ used in the simulation is larger than $N_{\text {cut }}$ appropriate for the fugacity of interest. Finally, $\beta W^{\mathrm{rev}}\left(z, N_{c}\right)$ evaluated using $p_{s}\left(z^{\prime}, N_{c}\right)$ should increase with $z^{\prime}$, reflecting a contribution from the configurations accounted for under the $N_{c}$-cluster with some of the molecules being more properly regarded as part of the vapor, since such configurations are energetically unfavorable and tend to increase the free energy of the $N_{c}$-cluster. The fact that $\beta W^{\text {rev }}\left(z, N_{c}\right)$ depends only negligibly on $z^{\prime}$ implies that the $V$ dependence of the thermodynamic properties of the $N_{c}$-cluster is also negligible as we claimed near the end of Sec. II B.

\section{CONCLUDING REMARKS}

We have presented a new approach in identifying a physical cluster. Our approach differs from the conventional ones in that no intermediate cluster such as the LBA cluster or the $i / v$ cluster is introduced, from which the physical clusters have to be isolated either by adjusting the parameter $v$ or by resorting to a rate theory. Thus the assumption implicit in the conventional approach that the physical clusters form a subset of these intermediate clusters is avoided. Our basic idea is to follow the stochastic evolution of a system while suppressing the fluctuations irrelevant to the nucleation event. In doing so, we demonstrate the utility of a grand canonical Monte Carlo simulation. Thus, the simulation preferentially samples the physical clusters without any prior knowledge regarding their detailed identity, and then directly determines their equilibrium distribution. The latter feature permits one to completely bypass the expensive free energy calculation; this in turn opens up the possibility of employing more realistic intermolecular potentials that have been hitherto computationally prohibitively expensive. With an efficient method in both identifying the physical clusters and determining their equilibrium distribution, one is now in a position to initiate a rate theory to capture the full molecular level details of the nucleation process.

\section{ACKNOWLEDGMENTS}

The authors wish to express gratitude to Professor Howard Reiss and his co-workers for the series of insightful papers (Refs. 1-6) and fruitful discussions, which led them to appreciate the importance of the physical clusters in the context of nucleation theory. This work was supported by National Science Foundation Grant No. ATM-9614105. Z.G.W. acknowledges support from the Camille and Henry Dreyfus Foundation, the Alfred P. Sloan Foundation, and the National Science Foundation. We also acknowledge support by a grant from the Mobil Corporation.

${ }^{1}$ H. Reiss, A. Tabazadeh, and J. Talbot, J. Chem. Phys. 92, 1266 (1990).

${ }^{2}$ H. M. Ellerby, C. L. Weakliem, and H. Riess, J. Chem. Phys. 95, 9209 (1991).

${ }^{3}$ H. M. Ellerby and H. Reiss, J. Chem. Phys. 97, 5766 (1992).

${ }^{4}$ C. L. Weakliem and H. Reiss, J. Chem. Phys. 99, 5374 (1993).

${ }^{5}$ C. L. Weakliem and H. Reiss, J. Chem. Phys. 101, 2398 (1994).

${ }^{6}$ K. J. Oh, X. C. Zeng, and H. Reiss, J. Chem. Phys. 107, 1242 (1997).

${ }^{7}$ Nucleation, edited by A. C. Zettlemoyer (Marcel Dekker, New York, 1969).

${ }^{8}$ J. K. Lee, J. A. Barker, and F. F. Abraham, J. Chem. Phys. 58, 3166 (1973).

${ }^{9}$ M. P. Allen and D. J. Tildesley, Computer Simulation of Liquids (Oxford University Press, New York, 1987).

${ }^{10}$ V. Talanquer and D. W. Oxtoby, J. Chem. Phys. 100, 5190 (1994).

${ }^{11}$ H. J. C. Berendsen, J. R. Grigera, and T. P. Straatsma, J. Phys. Chem. 91, 6269 (1987)

${ }^{12}$ K. Nishioka and G. M. Pound, Adv. Colloid Interface Sci. 7, 205 (1977).

${ }^{13}$ R. Kubo, Statistical Mechanics (Elsevier Science, Amsterdam, 1965).

${ }^{14}$ W. Band, J. Chem. Phys. 7, 324 (1939).

${ }^{15}$ W. Band, J. Chem. Phys. 7, 927 (1939).

${ }^{16}$ F. H. Stillinger, Jr., J. Chem. Phys. 38, 1486 (1963).

${ }^{17}$ D. Chandler, Introduction to Modern Statistical Mechanics (Oxford University Press, New York, 1987).

${ }^{18}$ R. Kelterbaum, N. Turki, A. Rahmouni, and E. Kochanski, J. Mol. Struct.: THEOCHEM 314, 191 (1994).

${ }^{19}$ Pictures of clusters were produced by a program RasMol developed by Dr. R. Sayle. 